\title{
Three two-dimensional Weyl steps in the circle problem I. The Hessian determinant
}

by

\author{
Ulrike M. A. Vorhauer and Eduard Wirsing (Ulm)
}

1. Summary. In a sequence of three papers we study the circle problem and its generalization involving the logarithmic mean. Most of the deeper results in this area depend on estimates of exponential sums. For the circle problem itself Chen has carried out such estimates using three two-dimensional Weyl steps with complicated techniques. We make the same Weyl steps but our approach is simpler and clearer. Crucial is a good understanding of the Hessian determinant that appears and a simple estimate of certain exponential integrals.

In Part I we determine the order of magnitude of the Hessian as well as that of the maximum of the second derivatives for the functions $h$, which are third order differences of the two-dimensional Euclidean vector norm.

2. Introduction. A number of questions connected with the circle problem depend on estimates for exponential sums of the type

$$
\sum_{\underline{x}} e(u\|\underline{x}\|) \quad \text { with } \underline{x}=(x, y) \in \mathbb{R}^{2},
$$

$u$ a real parameter, $e(x)=e^{2 \pi i x}$ and $\|\underline{x}\|=\sqrt{x^{2}+y^{2}}$, the Euclidean norm. One of the tools that have been successfully applied to this problem is the two-dimensional Weyl-van der Corput method, which originates with the important papers of Titchmarsh ([7]-[9]) followed by papers of Hua [2], Min [5], Richert [6], Chen Jingrun [1] and others. However, objections were raised against certain critical steps in the complicated proof by Walfisz [10] and Jarník [3]. The situation has apparently been corrected by subsequent papers of Min [5], Krätzel [4] etc. But even so, the paper of Chen, which is the one giving the best result along this line for the circle problem, is difficult to understand because of the enormous number of cases and subcases without an apparent idea.

1991 Mathematics Subject Classification: 11L, 11N. 
The object of the present paper is two-fold. On the one hand we wish to consider the logarithmic Riesz mean

$$
F_{\kappa}(x):=\frac{1}{\Gamma(\kappa+1)} \sum_{n<x} r_{2}(n) \log ^{\kappa}(x / n)
$$

rather than the unweighted sum $\sum_{n<x} r_{2}(n)$. A representation of $F_{\kappa}(x)$ that involves a truncated Voronoï type sum will be developed in Part II.

On the other hand, given the situation described above, we simplify and clarify the proof in two ways: Part I presents essentially a careful analysis of the Hessian in question. In fact Theorem A gives the precise order of its magnitude in simple terms. It turns out that the bad cases, where the Hessian is small, are so rare that we can replace the difficult Titchmarsh-Min estimate of the exponential integral by a somewhat weaker one, which has the advantage of being much simpler to formulate and easier to prove. Part III of our paper will present this theorem (Theorem 2) and the application to the Riesz mean. In particular we shall confirm Chen's exponent $\theta=12 / 37$ for $\kappa=0$.

After applying three two-dimensional Weyl steps we are left with sums of the type

$$
\sum_{\underline{x}} e(u h(\underline{x}, \underline{\underline{p}}))
$$

where $\underline{\underline{p}}=\left(\underline{p}_{1}, \underline{p}_{2}, \underline{p}_{3}\right), \underline{p}_{\nu}=\left(h_{\nu}, k_{\nu}\right)^{T}, h_{\nu}, k_{\nu} \in \mathbb{Z}$ and

$$
h(\underline{x}, \underline{\underline{p}})=\Delta_{\underline{p}} \Delta_{\underline{p}} \Delta_{\underline{p}}\|\underline{x}\|
$$

is the third order difference, using the definition $\Delta_{p} f(\underline{x})=f(\underline{x}+\underline{p})-f(\underline{x})$. Explicitly,

$$
h(\underline{x}, \underline{\underline{p}})=\sum_{\varepsilon_{1}, \varepsilon_{2}, \varepsilon_{3} \in\{0,1\}} \varepsilon_{1}^{\prime} \varepsilon_{2}^{\prime} \varepsilon_{3}^{\prime}\left\|\underline{x}+\varepsilon_{1} \underline{p}_{1}+\varepsilon_{2} \underline{p}_{2}+\varepsilon_{3} \underline{p}_{3}\right\|,
$$

where $\varepsilon_{\nu}^{\prime}=2 \varepsilon_{\nu}-1$ are \pm 1 , depending on whether $\varepsilon_{\nu}=1$ or 0 . Estimating these sums effectively requires knowledge of the size of the Hessian determinant $\left(h_{x x} h_{y y}-h_{x y}^{2}\right)(\underline{x}, \underline{p})$. The main subject of the present investigation is a careful analysis of the structure and size of this Hessian. Its basis is a homogeneity relation for $h$, which carries over to the Hessian determinant, and which is most easily expressed by replacing the vectors $\underline{x}$ and $\underline{p}{ }_{\nu}$ by complex numbers $z=x+i y$ and $p_{\nu}=h_{\nu}+i k_{\nu}$ respectively. Thus

$$
h\left(z, p_{1}, p_{2}, p_{3}\right)=\sum_{\varepsilon_{\nu} \in\{0,1\}} \varepsilon_{1}^{\prime} \varepsilon_{2}^{\prime} \varepsilon_{3}^{\prime} r\left(z+\varepsilon_{1} p_{1}+\varepsilon_{2} p_{2}+\varepsilon_{3} p_{3}\right),
$$

where $r(z)=|z|$. Obviously,

$$
h\left(c z, c p_{1}, c p_{2}, c p_{3}\right)=|c| h\left(z, p_{1}, p_{2}, p_{3}\right) \quad \text { for any } c \in \mathbb{C} .
$$


From this we derive in Lemma $1 \mathrm{~A}$ a corresponding relation for the Hessian $H$ of $h$, namely

$$
H\left(z, p_{1}, p_{2}, p_{3}\right)=\frac{1}{|z|^{2}} H\left(1, \frac{p_{1}}{z}, \frac{p_{2}}{z}, \frac{p_{3}}{z}\right),
$$

and then study the Taylor expansion of $H\left(1, q_{1}, q_{2}, q_{3}\right)$. The main result will be

THEOREM A. There is an absolute constant $\delta>0$ such that

$$
-H\left(1, q_{1}, q_{2}, q_{3}\right) \asymp\left|q_{1} q_{2} q_{3}\right|^{2} \max _{\nu=1,2,3}\left|\frac{\Im\left(q_{\nu}\right)}{q_{\nu}}\right|^{2}
$$

for any $q_{\nu} \in \mathbb{C} \backslash\{0\},\left|q_{\nu}\right| \leq \delta$, for $\nu=1,2,3$.

Since $\Im\left(q_{\nu}\right) /\left|q_{\nu}\right|=\sin \varphi_{\nu}$, where $\varphi_{\nu}$ is the angle between $z$ and $p_{\nu}$, $p_{\nu}=q_{\nu} z$, we may rewrite the theorem as

$$
-H\left(z, p_{1}, p_{2}, p_{3}\right) \asymp|z|^{-8}\left|p_{1} p_{2} p_{3}\right|^{2} \max _{\nu=1,2,3} \sin ^{2} \varphi_{\nu} .
$$

In particular the above Hessian vanishes if and only if each of the three complex numbers $p_{\nu}$ is a real multiple of $z$.

For our application, where in (1) we have to sum or integrate over $\underline{x}$ and to sum over $\underline{p}_{1}, \underline{p}_{2}, \underline{p}_{3}$, the theorem tells us that for any given $\underline{x}$ there are few values $\underline{p}_{1}, \underline{p}_{2}, \underline{p}_{3}$ that cause difficulties by making the Hessian small.

Also important for the treatment of the circle problem by the authors quoted above are lower estimates for the second partial derivatives of $h$. A good simultaneous measure for a lower as well as for an upper bound is

$$
Q\left(z, p_{1}, p_{2}, p_{3}\right):=\left(h_{x x}^{2}+2 h_{x y}^{2}+h_{y y}^{2}\right)\left(z, p_{1}, p_{2}, p_{3}\right) .
$$

This expression can be treated in a way that is remarkably similar to $H$, though somewhat easier. We prove

TheOREM B. There is an absolute constant $\delta>0$ such that

$$
Q\left(1, q_{1}, q_{2}, q_{3}\right) \asymp\left|q_{1} q_{2} q_{3}\right|^{2}
$$

for any $q_{\nu} \in \mathbb{C} \backslash\{0\},\left|q_{\nu}\right| \leq \delta(\nu=1,2,3)$. In particular,

$$
\max \left(\left|h_{x x}\right|,\left|h_{x y}\right|,\left|h_{y y}\right|\right) \asymp \frac{\left|p_{1} p_{2} p_{3}\right|}{|z|^{4}} .
$$

Actually we found that our treatment of the circle problem is also simpler in that it no longer needs a lower bound for these second derivatives. Nevertheless, we believe that in view of possible later improvements and as a result of independent interest Theorem $\mathrm{B}$, which comes along in a very natural way with the proof of Theorem A, should be included here.

A feature of Theorem A that contributes substantially to its value as well as to the difficulties of its proof is the following: If we develop $H(z, p)$ 
in its Taylor series with respect to the real and imaginary parts of the $p_{\nu}$, then the leading form, actually the one of order six, is only semidefinite but nevertheless it determines the absolute size and sign of $H(z, p)$ uniformly for all $p_{\nu}$ that are small compared to $z$. In $Q(z, p)$, by contrast, the leading sixth order form is positive definite and the proof of Theorem B correspondingly easier.

In retrospect the background of the papers of Titchmarsh, Hua and Min becomes clear. Each of them uses three Weyl steps. In Titchmarsh's paper [8] only one is two-dimensional; the two one-dimensional steps are taken along the $x$-axis. By the obvious symmetry of the circle problem, he can restrict summation to an octant adjacent to the $y$-axis and therefore has $\max _{\nu} \varphi_{\nu} \geq \pi / 4$ and

$$
-H(\underline{x}, \underline{\underline{p}}) \gg\|\underline{x}\|^{-8}\left\|\left(h_{1}, 0\right)\right\|^{2}\left\|\left(h_{2}, 0\right)\right\|^{2}\left\|\left(h_{3}, k_{3}\right)\right\|^{2}
$$

(see $\S 11(4)$ of that paper). We may add that the restriction to the above octant would have been unnecessary if he had taken the one-dimensional Weyl steps in different directions. On the other hand this restriction has the effect that $\max \varphi_{\nu} \geq \pi / 4$ already if only one Weyl step is taken along the $x$-axis. This then is the basis of Hua's paper, who thereby is able to use two two-dimensional Weyl steps. His lower bound for the Hessian

$$
-H(\underline{x}, \underline{p}) \gg\|\underline{x}\|^{-8}\left(X_{1}^{2}+X_{2}^{2}+X_{3}^{2}\right)
$$

is easily seen to be

$$
\gg\|\underline{x}\|^{-8}\left\|\left(h_{1}, 0\right)\right\|^{2}\left\|\left(h_{2}, k_{2}\right)\right\|^{2}\left\|\left(h_{3}, k_{3}\right)\right\|^{2},
$$

which corresponds to Theorem A in this case. If one employs all three Weyl steps in the two-dimensional form, as Chen does, it is no longer possible to avoid small values of the Hessian by an appeal to symmetry. Furthermore Chen's estimate of the Hessian (Lemma 1) is not comparable to our Theorem A. Both aspects contribute substantially to the extreme technical difficulties of his paper.

3. Sketch of proofs. Both proofs proceed essentially in four steps:

1. Formulation of homogeneity relations for the functions $H\left(z, p_{1}, p_{2}, p_{3}\right)$ and $Q\left(z, p_{1}, p_{2}, p_{3}\right)$. This reduces further analysis to the study of

$$
H\left(1, q_{1}, q_{2}, q_{3}\right), \quad p_{\nu}=q_{\nu} z, \quad q_{\nu}=\eta_{\nu}+i \kappa_{\nu}, \quad \eta_{\nu}, \kappa_{\nu} \in \mathbb{R} .
$$

2. Taylor expansion of $H\left(1, q_{1}, q_{2}, q_{3}\right)$ and $Q\left(1, q_{1}, q_{2}, q_{3}\right)$ in terms of the $\eta_{\nu}, \kappa_{\nu}$. Both functions vanish at the origin with order six. Thus, collecting all terms of order $n$ in $H_{n}, Q_{n}$ respectively, we have

$$
H\left(1, q_{1}, q_{2}, q_{3}\right)=\sum_{n=6}^{\infty} H_{n} \quad \text { and } \quad Q\left(1, q_{1}, q_{2}, q_{3}\right)=\sum_{n=6}^{\infty} Q_{n} .
$$


Since $H\left(1, q_{1}, q_{2}, q_{3}\right)$ and $Q\left(1, q_{1}, q_{2}, q_{3}\right)$ are symmetric in $q_{1}, q_{2}, q_{3}$, the forms $H_{6}, Q_{6}$ are symmetric functions of the pairs $\left(\eta_{\nu}, \kappa_{\nu}\right)$. Thus they can be expanded in terms of the homogenized elementary symmetric functions, i.e. of the coefficients $X_{i}$ of

$$
\prod_{\nu=1}^{3}\left(\eta_{\nu} u+\kappa_{\nu} v\right)=\sum_{i=0}^{3} X_{i} u^{i} v^{3-i}
$$

3. Due to the fact that this ternary form has only real zeros, the variables $X_{\nu}$ do not behave independently. Therefore between functions of $X_{0}, \ldots, X_{3}$ there may hold inequalities that are not obvious. Thus, sacrificing some accuracy, we can simplify $H_{6}, Q_{6}$ into the more convenient expressions $\widetilde{H} \asymp H_{6}$ and $\widetilde{Q} \asymp Q_{6}$ which form the right hand sides in Theorem A and Theorem B.

4. Proving that $\widetilde{H}$ and $\widetilde{Q}$ dominate the two remainders $\sum_{n=7}^{\infty} H_{n}$ and $\sum_{n=7}^{\infty} Q_{n}$ respectively, thereby globalizing the estimates that so far were only infinitesimal.

4. Homogeneity relations. If we denote by $\mathcal{H}_{z}(z=x+i y)$ the operator which generates the Hessian $\mathcal{H}_{z} f=f_{x x} f_{y y}-f_{x y}^{2}$ with respect to $x$ and $y$, the following chain rule holds:

Lemma 1A. Let $w(z)=u(z)+i v(z)$ and assume that $u_{x}, u_{y}, v_{x}, v_{y}$ are constant. Then

$$
\mathcal{H}_{z} f(w(z))=\left|\begin{array}{ll}
u_{x} & u_{y} \\
v_{x} & v_{y}
\end{array}\right|^{2} \mathcal{H}_{w} f(w) .
$$

In particular for any $c \in \mathbb{C}$,

$$
\mathcal{H}_{z} f(c z)=\left.|c|^{4} \mathcal{H}_{w} f(w)\right|_{w=c z} .
$$

Proof. To simplify the proof we write $x_{1}, x_{2}$ and $u_{1}, u_{2}$ for $x, y$ and $u$, $v$ respectively. The chain rule

$$
\frac{\partial}{\partial x_{i}}=\sum_{k} \frac{\partial u_{k}}{\partial x_{i}} \frac{\partial}{\partial u_{k}}
$$

yields, since $\partial u_{l} / \partial x_{i}$ are constant,

$$
\begin{aligned}
\frac{\partial^{2} f}{\partial x_{i} \partial x_{j}} & =\left(\sum_{k} \frac{\partial u_{k}}{\partial x_{i}} \frac{\partial}{\partial u_{k}}\right)\left(\sum_{l} \frac{\partial u_{l}}{\partial x_{j}} \frac{\partial}{\partial u_{l}}\right) f \\
& =\sum_{k, l} \frac{\partial u_{k}}{\partial x_{i}} \frac{\partial u_{l}}{\partial x_{j}} \frac{\partial^{2} f}{\partial u_{k} \partial u_{l}} .
\end{aligned}
$$

The multiplication theorem for determinants gives (3). In the special case 
$w=c z$ we have

$$
\left|\begin{array}{ll}
u_{x} & u_{y} \\
v_{x} & v_{y}
\end{array}\right|=\left|\begin{array}{cc}
a & -b \\
b & a
\end{array}\right|=a^{2}+b^{2}=|c|^{2} .
$$

Lemma 2A. If $f$ is a function of $z$ and some parameters $p_{1}, \ldots, p_{r} \in \mathbb{C}$, that with $p=\left(p_{1}, \ldots, p_{r}\right)$ satisfies a relation

$$
f(c z, c p)=|c| f(z, p) \quad \text { for any } c \in \mathbb{C},
$$

and if we put $H(z, p):=\mathcal{H}_{z} f(z, p)$, then

$$
H(c z, c p)=\frac{1}{|c|^{2}} H(z, p) .
$$

In particular

$$
H(z, p)=\frac{1}{|z|^{2}} H\left(1, \frac{p}{z}\right) .
$$

Pr o o f. If $\mathcal{H}_{z}$ is applied to (5), then on the one hand according to Lemma $1 \mathrm{~A}$ we have

$$
\mathcal{H}_{z} f(c z, c p)=|c|^{4} H(c z, c p),
$$

and on the other

$$
\mathcal{H}_{z}(|c| f(z, p))=|c|^{2} \mathcal{H}_{z} f(z, p)=|c|^{2} H(z, p) .
$$

The lemma applies in particular to the function $h$ given in (2) and its Hessian $H(z, p)$.

In comparison to Lemma $1 \mathrm{~A}$ the following lemma is somewhat narrower. In contrast to Lemma $1 \mathrm{~A}$ it cannot be proved for general affine mappings $w$, but only for similarities $w=c z$.

Lemma 1B. Let $\mathcal{Q}_{z}, z=x+i y$, denote the operator defined by

$$
\mathcal{Q}_{z} f:=f_{x x}^{2}+2 f_{x y}^{2}+f_{y y}^{2} .
$$

Then for $c \in \mathbb{C}$,

$$
\mathcal{Q}_{z} f(c z)=\left.|c|^{4} \mathcal{Q}_{w} f(w)\right|_{w=c z} .
$$

Proof. With the notation of Lemma 1A, putting $c=a+i b$, we have $u_{i}=\sum_{j} t_{i j} x_{j}$ with the matrix $T=\left(t_{i j}\right)=\left(\begin{array}{cc}a & -b \\ b & a\end{array}\right)$. Consequently, by (4),

$$
\frac{\partial^{2} f}{\partial x_{i} \partial x_{j}}=\sum_{k, l} t_{k i} t_{l j} \frac{\partial^{2} f}{\partial u_{k} \partial u_{l}} \text {. }
$$

From the orthogonality of $T, \sum_{i} t_{k i} t_{m i}=\left(a^{2}+b^{2}\right) \delta_{k m}$, we obtain

$$
\sum_{i, j}\left(\frac{\partial^{2} f}{\partial x_{i} \partial x_{j}}\right)^{2}=\sum_{i, j}\left\{\sum_{k, l} t_{k i} t_{l j} \frac{\partial^{2} f}{\partial u_{k} \partial u_{l}}\right\}\left\{\sum_{m, n} t_{m i} t_{n j} \frac{\partial^{2} f}{\partial u_{m} \partial u_{n}}\right\}
$$




$$
\begin{aligned}
& =\sum_{k, l, m, n}\left\{\sum_{i} t_{k i} t_{m i}\right\}\left\{\sum_{j} t_{l j} t_{n j}\right\} \frac{\partial^{2} f}{\partial u_{k} \partial u_{l}} \frac{\partial^{2} f}{\partial u_{m} \partial u_{n}} \\
& =\left(a^{2}+b^{2}\right)^{2} \sum_{k, l}\left(\frac{\partial^{2} f}{\partial u_{k} \partial u_{l}}\right)^{2} \cdot \boldsymbol{}
\end{aligned}
$$

From this the next lemma follows in the same way as Lemma $2 \mathrm{~A}$ from Lemma 1A.

Lemma 2B. Let $f$ satisfy a relation (5) as in Lemma $2 \mathrm{~A}$ and define $Q(z, p):=\mathcal{Q}_{z} f(z, p)$. Then for any $c \in \mathbb{C}$,

$$
Q(c z, c p)=\frac{1}{|c|^{2}} Q(z, p) \text {. }
$$

In particular

$$
Q(z, p)=\frac{1}{|z|^{2}} Q\left(1, \frac{p}{z}\right)
$$

5. Taylor expansions. Next we study the Taylor expansion of the function

(6) $h(z, p)=\Delta_{p_{1}} \Delta_{p_{2}} \Delta_{p_{3}} r(z)=\sum_{\varepsilon_{\nu} \in\{0,1\}} \varepsilon_{1}^{\prime} \varepsilon_{2}^{\prime} \varepsilon_{3}^{\prime} r\left(z+\varepsilon_{1} p_{1}+\varepsilon_{2} p_{2}+\varepsilon_{3} p_{3}\right)$,

more precisely those of its derivatives $h_{x x}, h_{x y}$ and $h_{y y}$ at $z=1$. Here $z=x+i y, r(z)=|z|=\sqrt{x^{2}+y^{2}}$ and $p=\left(p_{1}, p_{2}, p_{3}\right), p_{\nu}=z q_{\nu}$ with $q_{\nu}=\eta_{\nu}+i \kappa_{\nu}\left(\eta_{\nu}, \kappa_{\nu} \in \mathbb{R}\right)$. In this connection we denote by $\mathcal{O}_{n}$ any remainder terms of order $\geq n$ in $\eta_{1}, \ldots, \kappa_{3}$. Further we write

$$
\begin{gathered}
X_{0}=\kappa_{1} \kappa_{2} \kappa_{3}, \quad X_{1}=\kappa_{1} \kappa_{2} \eta_{3}+\kappa_{1} \eta_{2} \kappa_{3}+\eta_{1} \kappa_{2} \kappa_{3}, \\
X_{2}=\kappa_{1} \eta_{2} \eta_{3}+\eta_{1} \kappa_{2} \eta_{3}+\eta_{1} \eta_{2} \kappa_{3}, \quad X_{3}=\eta_{1} \eta_{2} \eta_{3}
\end{gathered}
$$

for the elementary symmetric functions of $\left(\eta_{\nu}, \kappa_{\nu}\right), \nu=1,2,3$.

Lemma 3. The second partial derivatives have representations

$$
\begin{aligned}
& h_{x x}(1, q)=-6 X_{1}+\mathcal{O}_{4}, \\
& h_{x y}(1, q)=9 X_{0}-6 X_{2}+\mathcal{O}_{4}, \\
& h_{y y}(1, q)=9 X_{1}-6 X_{3}+\mathcal{O}_{4} .
\end{aligned}
$$

Proof. In (6) let us abbreviate $\sum_{\nu=1}^{3} \varepsilon_{\nu} p_{\nu}$ by $\varepsilon p$, an inner product. Thus

$$
h(1, q)=\sum_{\varepsilon_{\nu}} \varepsilon_{1}^{\prime} \varepsilon_{2}^{\prime} \varepsilon_{3}^{\prime} r(1+\varepsilon q)
$$

and therefore

$$
h_{x x}(1, q)=\sum_{\varepsilon_{\nu}} \varepsilon_{1}^{\prime} \varepsilon_{2}^{\prime} \varepsilon_{3}^{\prime} r_{x x}(1+\varepsilon q)
$$


etc. Since $r_{x x}=y^{2} / r^{3}, r_{x y}=-x y / r^{3}$ and $r_{y y}=x^{2} / r^{3}$, we obtain

$$
\begin{aligned}
& h_{x x}(1, q)=\sum_{\varepsilon_{\nu}} \varepsilon_{1}^{\prime} \varepsilon_{2}^{\prime} \varepsilon_{3}^{\prime} \frac{(\varepsilon \kappa)^{2}}{|1+\varepsilon q|^{3}}, \\
& h_{x y}(1, q)=-\sum_{\varepsilon_{\nu}} \varepsilon_{1}^{\prime} \varepsilon_{2}^{\prime} \varepsilon_{3}^{\prime} \frac{(1+\varepsilon \eta) \varepsilon \kappa}{|1+\varepsilon q|^{3}}, \\
& h_{y y}(1, q)=\sum_{\varepsilon_{\nu}} \varepsilon_{1}^{\prime} \varepsilon_{2}^{\prime} \varepsilon_{3}^{\prime} \frac{(1+\varepsilon \eta)^{2}}{|1+\varepsilon q|^{3}} .
\end{aligned}
$$

Expanding $|1+\varepsilon q|^{-3}$ as follows:

$$
\begin{aligned}
\frac{1}{|1+\varepsilon q|^{3}} & =\left((1+\varepsilon \eta)^{2}+(\varepsilon \kappa)^{2}\right)^{-3 / 2} \\
& =(1+\varepsilon \eta)^{-3}\left(1+\frac{(\varepsilon \kappa)^{2}}{(1+\varepsilon \eta)^{2}}\right)^{-3 / 2} \\
& =\sum_{m \in \mathbb{N}_{0}}\left(\begin{array}{c}
-3 / 2 \\
m
\end{array}\right)(\varepsilon \kappa)^{2 m}(1+\varepsilon \eta)^{-2 m-3},
\end{aligned}
$$

we get

(9)

$$
\begin{aligned}
r_{x x}(1+\varepsilon q) & =\sum_{m \in \mathbb{N}_{0}}\left(\begin{array}{c}
-3 / 2 \\
m
\end{array}\right)(\varepsilon \kappa)^{2 m+2}(1+\varepsilon \eta)^{-2 m-3} \\
& =\sum_{m, n \in \mathbb{N}_{0}}\left(\begin{array}{c}
-3 / 2 \\
m
\end{array}\right)\left(\begin{array}{c}
-2 m-3 \\
n
\end{array}\right)(\varepsilon \kappa)^{2 m+2}(\varepsilon \eta)^{n}, \\
r_{x y}(1+\varepsilon q) & =-\sum_{m, n \in \mathbb{N}_{0}}\left(\begin{array}{c}
-3 / 2 \\
m
\end{array}\right)\left(\begin{array}{c}
-2 m-2 \\
n
\end{array}\right)(\varepsilon \kappa)^{2 m+1}(\varepsilon \eta)^{n}, \\
r_{y y}(1+\varepsilon q) & =\sum_{m, n \in \mathbb{N}_{0}}\left(\begin{array}{c}
-3 / 2 \\
m
\end{array}\right)\left(\begin{array}{c}
-2 m-1 \\
n
\end{array}\right)(\varepsilon \kappa)^{2 m}(\varepsilon \eta)^{n} .
\end{aligned}
$$

The Taylor expansions of $h_{x x}(1, q)$ etc. follow by summation over $\varepsilon_{\nu}$ according to formula (7). As will be seen, the terms of order $\leq 2$ vanish:

$$
\sum_{\varepsilon_{\nu}} \varepsilon_{1}^{\prime} \varepsilon_{2}^{\prime} \varepsilon_{3}^{\prime}(\varepsilon \eta)^{k}(\varepsilon \kappa)^{l}=0 \quad \text { if } k+l \leq 2
$$

The reason is that, after multiplying out $(\varepsilon \eta)^{k}$ and $(\varepsilon \kappa)^{l}$, in each of the terms that arise, one of the variables $\varepsilon_{\nu}$ is missing and summation over this $\varepsilon_{\nu}$ gives $\sum_{\varepsilon_{\nu}} \varepsilon_{\nu}^{\prime}=1-1=0$. The terms of order three $(k+l=3)$ lead to the symmetric functions $X_{0}, \ldots, X_{3}$. As before summation over the $\varepsilon_{\nu}$ eliminates all terms in which at least one $\varepsilon_{\nu}$ is missing, and in the rest only 
the value 1 for each variable $\varepsilon_{\nu}$ contributes. What remains is

$$
\begin{gathered}
\sum_{\varepsilon_{\nu}} \varepsilon_{1}^{\prime} \varepsilon_{2}^{\prime} \varepsilon_{3}^{\prime}(\varepsilon \kappa)^{3}=6 \kappa_{1} \kappa_{2} \kappa_{3}=6 X_{0}, \\
\sum_{\varepsilon_{\nu}} \varepsilon_{1}^{\prime} \varepsilon_{2}^{\prime} \varepsilon_{3}^{\prime}(\varepsilon \kappa)^{2}(\varepsilon \eta)=2\left(\kappa_{1} \kappa_{2} \eta_{3}+\kappa_{1} \kappa_{3} \eta_{2}+\kappa_{2} \kappa_{3} \eta_{1}\right)=2 X_{1}
\end{gathered}
$$

and symmetrically

$$
\sum_{\varepsilon_{\nu}} \varepsilon_{1}^{\prime} \varepsilon_{2}^{\prime} \varepsilon_{3}^{\prime}(\varepsilon \kappa)(\varepsilon \eta)^{2}=2 X_{2}, \quad \sum_{\varepsilon_{\nu}} \varepsilon_{1}^{\prime} \varepsilon_{2}^{\prime} \varepsilon_{3}^{\prime}(\varepsilon \eta)^{3}=6 X_{3} .
$$

Thus we find the term of order three in the expansion of $h_{x x}$ from (7) and (9) with $2 m+2+n=3$, that is, with $m=0$ and $n=1$, as

$$
\left(\begin{array}{c}
-3 / 2 \\
0
\end{array}\right)\left(\begin{array}{c}
-3 \\
1
\end{array}\right) \sum_{\varepsilon_{\nu}} \varepsilon_{1}^{\prime} \varepsilon_{2}^{\prime} \varepsilon_{3}^{\prime}(\varepsilon \kappa)^{2} \varepsilon \eta=-6 X_{1} .
$$

Similarly for $h_{x y}$ we have to evaluate the contributions from the terms with $2 m+1+n=3$, that is, from $m=0, n=2$ and $m=1, n=0$ :

$$
\begin{array}{r}
-\left(\left(\begin{array}{c}
-3 / 2 \\
0
\end{array}\right)\left(\begin{array}{c}
-2 \\
2
\end{array}\right) \sum_{\varepsilon_{\nu}} \varepsilon_{1}^{\prime} \varepsilon_{2}^{\prime} \varepsilon_{3}^{\prime}(\varepsilon \kappa)(\varepsilon \eta)^{2}+\left(\begin{array}{c}
-3 / 2 \\
1
\end{array}\right)\left(\begin{array}{c}
-4 \\
0
\end{array}\right) \sum_{\varepsilon_{\nu}} \varepsilon_{1}^{\prime} \varepsilon_{2}^{\prime} \varepsilon_{3}^{\prime}(\varepsilon \kappa)^{3}\right) \\
=-6 X_{2}+9 X_{0},
\end{array}
$$

and finally for $h_{y y}$ it is $2 m+n=3$, that is, $m=0, n=3$ and $m=n=1$ :

$$
\begin{array}{r}
\left(\begin{array}{c}
-3 / 2 \\
0
\end{array}\right)\left(\begin{array}{c}
-1 \\
3
\end{array}\right) \sum_{\varepsilon_{\nu}} \varepsilon_{1}^{\prime} \varepsilon_{2}^{\prime} \varepsilon_{3}^{\prime}(\varepsilon \eta)^{3}+\left(\begin{array}{c}
-3 / 2 \\
1
\end{array}\right)\left(\begin{array}{c}
-3 \\
1
\end{array}\right) \sum_{\varepsilon_{\nu}} \varepsilon_{1}^{\prime} \varepsilon_{2}^{\prime} \varepsilon_{3}^{\prime}(\varepsilon \kappa)^{2} \varepsilon \eta \\
=-6 X_{3}+9 X_{1} .
\end{array}
$$

Lemma 4. We have $H(1, q)=H_{6}+\mathcal{O}_{7}$, where

$$
H_{6}=-9\left(9 X_{0}^{2}+6 X_{1}^{2}+4 X_{2}^{2}-12 X_{0} X_{2}-4 X_{1} X_{3}\right),
$$

and $Q(1, q)=Q_{6}+\mathcal{O}_{7}$, where

$$
Q_{6}=9\left(18 X_{0}^{2}+13 X_{1}^{2}+8 X_{2}^{2}+4 X_{3}^{2}-24 X_{0} X_{2}-12 X_{1} X_{3}\right) .
$$

Proof. This follows by inserting the result of Lemma 3 into $H(1, q)=$ $\left(h_{x x} h_{y y}-h_{x y}^{2}\right)(1, q)$ and $Q(1, q)=\left(h_{x x}^{2}+2 h_{x y}^{2}+h_{y y}^{2}\right)(1, q)$ respectively.

In the next step we show how the quadratic forms $H_{6}$ and $Q_{6}$ can be estimated from below and above by simpler and more informative expressions.

Lemma 5. Let

$$
\widetilde{H}=\widetilde{H}(q)=\sum_{\nu=1}^{3}\left(\Im q_{\nu}\right)^{2} \prod_{\mu \neq \nu}\left|q_{\mu}\right|^{2} \quad \text { and } \quad \widetilde{Q}=\widetilde{Q}(q)=\left|q_{1} q_{2} q_{3}\right|^{2} .
$$


Then

$$
2 \widetilde{H} \leq-\frac{1}{9} H_{6} \leq 8 \widetilde{H} \quad \text { and } \quad 4 \widetilde{Q} \leq \frac{1}{9} Q_{6} \leq 18 \widetilde{Q} .
$$

Proof. We note that the $X_{i} / X_{0}$ are the elementary symmetric functions of the $\alpha_{\nu}=\eta_{\nu} / \kappa_{\nu}$ :

$$
X_{i}=\sigma_{i}(\alpha) X_{0} \quad \text { for } i=1,2,3, \quad X_{0}=\kappa_{1} \kappa_{2} \kappa_{3}, \quad \alpha=\left(\alpha_{1}, \alpha_{2}, \alpha_{3}\right) .
$$

Thus by Lemma 4,

$$
\frac{-1}{9 X_{0}^{2}} H_{6}=9+6 \sigma_{1}^{2}+4 \sigma_{2}^{2}-12 \sigma_{2}-4 \sigma_{1} \sigma_{3} .
$$

We use the simple identities

$$
\begin{aligned}
& \sigma_{1}^{2}=\sigma_{1}\left(\alpha_{\nu}^{2}\right)+2 \sigma_{2}, \\
& \sigma_{2}^{2}=\sigma_{2}\left(\alpha_{\nu}^{2}\right)+2 \sigma_{1} \sigma_{3},
\end{aligned}
$$

that follow from squaring the defining equations $\sigma_{1}=\alpha_{1}+\alpha_{2}+\alpha_{3}$ and $\sigma_{2}=\alpha_{1} \alpha_{2}+\alpha_{2} \alpha_{3}+\alpha_{3} \alpha_{1}$, and we obtain

$$
\frac{-1}{9 X_{0}^{2}} H_{6}=9+6 \sigma_{1}\left(\alpha_{\nu}^{2}\right)+4 \sigma_{2}\left(\alpha_{\nu}^{2}\right)+4 \sigma_{1} \sigma_{3}
$$

Since $2 \sigma_{1} \sigma_{3} \geq-\sigma_{2}\left(\alpha_{\nu}^{2}\right)$ by (11) and obviously $\sigma_{1}\left(\alpha_{\nu}^{2}\right) \geq 0, \sigma_{2}\left(\alpha_{\nu}^{2}\right) \geq 0$, we may continue with

$$
\begin{aligned}
\frac{-1}{9 X_{0}^{2}} H_{6} & \geq 9+6 \sigma_{1}\left(\alpha_{\nu}^{2}\right)+2 \sigma_{2}\left(\alpha_{\nu}^{2}\right) \\
& \geq 2\left(3+2 \sigma_{1}\left(\alpha_{\nu}^{2}\right)+\sigma_{2}\left(\alpha_{\nu}^{2}\right)\right) \\
& =2\left(\left(1+\alpha_{1}^{2}\right)\left(1+\alpha_{2}^{2}\right)+\left(1+\alpha_{1}^{2}\right)\left(1+\alpha_{3}^{2}\right)+\left(1+\alpha_{2}^{2}\right)\left(1+\alpha_{3}^{2}\right)\right),
\end{aligned}
$$

which is

$$
-\frac{1}{9} H_{6} \geq 2\left(\kappa_{1}^{2}\left|q_{2} q_{3}\right|^{2}+\kappa_{2}^{2}\left|q_{1} q_{3}\right|^{2}+\kappa_{3}^{2}\left|q_{1} q_{2}\right|^{2}\right)=2 \widetilde{H}(q) .
$$

For the opposite inequality we use

$$
\begin{aligned}
2 \sigma_{1} \sigma_{3} & =\alpha_{1}^{2}\left(2 \alpha_{2} \alpha_{3}\right)+\alpha_{2}^{2}\left(2 \alpha_{3} \alpha_{1}\right)+\alpha_{3}^{2}\left(2 \alpha_{1} \alpha_{2}\right) \\
& \leq \alpha_{1}^{2}\left(\alpha_{2}^{2}+\alpha_{3}^{2}\right)+\alpha_{2}^{2}\left(\alpha_{3}^{2}+\alpha_{1}^{2}\right)+\alpha_{3}^{2}\left(\alpha_{1}^{2}+\alpha_{2}^{2}\right) \\
& =2\left(\alpha_{1}^{2} \alpha_{2}^{2}+\alpha_{2}^{2} \alpha_{3}^{2}+\alpha_{3}^{2} \alpha_{1}^{2}\right) \\
2 \sigma_{1} \sigma_{3} & \leq 2 \sigma_{2}\left(\alpha_{\nu}^{2}\right)
\end{aligned}
$$

and find

$$
\frac{-1}{9 X_{0}^{2}} H_{6} \leq 9+6 \sigma_{1}\left(\alpha_{\nu}^{2}\right)+8 \sigma_{2}\left(\alpha_{\nu}^{2}\right) \leq 8\left(3+2 \sigma_{1}\left(\alpha_{\nu}^{2}\right)+\sigma_{2}\left(\alpha_{\nu}^{2}\right)\right) ;
$$

hence

$$
-\frac{1}{9} H_{6} \leq 8 \widetilde{H}(q)
$$


We turn to $Q(1, q)$. Here Lemma 4 gives

$$
\begin{aligned}
\frac{1}{9 X_{0}^{2}} Q_{6} & =18+13 \sigma_{1}^{2}+8 \sigma_{2}^{2}+4 \sigma_{3}^{2}-24 \sigma_{2}-12 \sigma_{1} \sigma_{3} \\
& =18+13 \sigma_{1}\left(\alpha_{\nu}^{2}\right)+8 \sigma_{2}\left(\alpha_{\nu}^{2}\right)+4 \sigma_{3}\left(\alpha_{\nu}^{2}\right)+2 \sigma_{2}+4 \sigma_{1} \sigma_{3} .
\end{aligned}
$$

Concerning the lower bound, we get $2 \sigma_{2} \geq-\sigma_{1}\left(\alpha_{\nu}^{2}\right)$ and $2 \sigma_{1} \sigma_{3} \geq-\sigma_{2}\left(\alpha_{\nu}^{2}\right)$ by (10) and (11) and therefore

$$
\begin{aligned}
\frac{1}{9 X_{0}^{2}} Q_{6} & \geq 18+12 \sigma_{1}\left(\alpha_{\nu}^{2}\right)+6 \sigma_{2}\left(\alpha_{\nu}^{2}\right)+4 \sigma_{3}\left(\alpha_{\nu}^{2}\right) \\
& \geq 4\left(1+\alpha_{1}^{2}\right)\left(1+\alpha_{2}^{2}\right)\left(1+\alpha_{3}^{2}\right) .
\end{aligned}
$$

The upper bound follows with $\sigma_{2} \leq \sigma_{1}\left(\alpha_{\nu}^{2}\right)$ and (12) from (13):

$$
\begin{aligned}
\frac{1}{9 X_{0}^{2}} Q_{6} & \leq 18+15 \sigma_{1}\left(\alpha_{\nu}^{2}\right)+12 \sigma_{2}\left(\alpha_{\nu}^{2}\right)+4 \sigma_{3}\left(\alpha_{\nu}^{2}\right) \\
& \leq 18\left(1+\alpha_{1}^{2}\right)\left(1+\alpha_{2}^{2}\right)\left(1+\alpha_{3}^{2}\right) .
\end{aligned}
$$

\section{Globalizing the estimates}

LEMma 6. We have

$$
\begin{aligned}
& H(1, q)=H_{6}+O\left(\widetilde{H}\left(\left|q_{1}\right|+\left|q_{2}\right|+\left|q_{3}\right|\right)\right), \\
& Q(1, q)=Q_{6}+O\left(\widetilde{Q}\left(\left|q_{1}\right|+\left|q_{2}\right|+\left|q_{3}\right|\right)\right) .
\end{aligned}
$$

Proof. Writing $h_{x x}(1, q)$ as $\Delta_{q_{1}}\left(\Delta_{q_{2}} \Delta_{q_{3}} r_{x x}\right)$ makes it obvious that $h_{x x}\left(1,0, q_{2}, q_{3}\right)=0$, similarly for $h_{x y}$ and $h_{y y}$. Therefore each monomial

$$
\eta_{1}^{i_{1}} \eta_{2}^{i_{2}} \eta_{3}^{i_{3}} \kappa_{1}^{j_{1}} \kappa_{2}^{j_{2}} \kappa_{3}^{j_{3}}
$$

that appears in the Taylor expansion of $h_{x x}, h_{x y}$ or $h_{y y}$ has a divisor $\eta_{1}$ or $\kappa_{1}$, i.e. $i_{1}+j_{1} \geq 1$. Therefore, monomials appearing in $H(1, q)$ or $Q(1, q)$ have $i_{1}+j_{1} \geq 2$, as they are both quadratic in $h_{x x}, h_{x y}$ and $h_{y y}$. Similarly $i_{2}+j_{2} \geq 2$ and $i_{3}+j_{3} \geq 2$. Let us, in the first instance, consider a monomial $M$ that appears in $Q(1, q)$ and which therefore has $i_{\nu}+j_{\nu} \geq 2$ for $\nu=1,2,3$. If we replace any $\eta_{\nu} \kappa_{\nu}$ that may occur by $\frac{1}{2}\left(\eta_{\nu}^{2}+\kappa_{\nu}^{2}\right)$ then up to eight new monomials arise that together bound our original monomial, and all of them have $i_{\nu} \geq 2$ or $j_{\nu} \geq 2$ for $\nu=1,2,3$. Such monomials, if they are of order six, are summands of $\widetilde{Q}$, which implies $M \leq \widetilde{Q}$. Any $M$ of higher order has at least one extra factor $\eta_{\nu}$ or $\kappa_{\nu}$ and hence is $\ll \widetilde{Q}\left(\left|q_{1}\right|+\left|q_{2}\right|+\left|q_{3}\right|\right)$. This proves (B).

Part (A) is a bit more tricky. We note first that the summands of $\widetilde{H}$ are exactly those monomials which have no elements other than 0 or 2 in their matrix of exponents

$$
\left(\begin{array}{lll}
i_{1} & i_{2} & i_{3} \\
j_{1} & j_{2} & j_{3}
\end{array}\right)
$$


and where each column as well as the second line contains at least one 2 , while $i_{1}+\ldots+j_{3}=6$.

On the other hand a monomial $M$ from $H(1, q)=h_{x x} h_{y y}-h_{x y}^{2}$ fulfills $i_{\nu}+j_{\nu} \geq 2$ for each $\nu$, as we have seen. But moreover now we have $j_{1}+j_{2}+$ $j_{3} \geq 2$, as a consequence of the terms $(\varepsilon \kappa)^{2}$ and $\varepsilon \kappa$ in the representations (8) of $h_{x x}, h_{x y}$ and $h_{y y}$ respectively. Any $M$ of this type has a divisor $T$ for which $i_{\nu}+j_{\nu}=2$ for each $\nu=1,2,3$ and $j_{1}+j_{2}+j_{3} \geq 2$, since equality can be obtained in each column by reducing just $i_{\nu}$ unless $j_{\nu} \geq 3$, in which case we replace $j_{\nu}$ by 2 .

If all $j_{\nu}$ are not 1 , and thus even, $T$ is one of the summands of $\widetilde{H}$. Otherwise, apart from order, we have $i_{1}=j_{1}=1$ and $\left(i_{2}=0, j_{2}=2\right.$ or $i_{2}=j_{2}=1$ ) and in any case $j_{3}$ may or may not be equal to 1 . In the first case we apply

as before, in the second case

$$
\left|\eta_{1} \kappa_{1}\right| \leq \frac{1}{2}\left(\eta_{1}^{2}+\kappa_{1}^{2}\right)
$$

$$
\left|\eta_{1} \kappa_{1} \eta_{2} \kappa_{2}\right| \leq \frac{1}{2}\left(\left(\eta_{1} \kappa_{2}\right)^{2}+\left(\eta_{2} \kappa_{1}\right)^{2}\right)
$$

If there is a factor $\eta_{3} \kappa_{3}$, we replace it too by $\frac{1}{2}\left(\eta_{3}^{2}+\kappa_{3}^{2}\right)$, so that in any case

$$
|T| \leq\left(\eta_{1}^{2}+\kappa_{1}^{2}\right) \kappa_{2}^{2}\left(\eta_{3}^{2}+\kappa_{3}^{2}\right) \leq \widetilde{H} \quad \text { or } \quad|T| \leq\left(\eta_{1}^{2} \kappa_{2}^{2}+\eta_{2}^{2} \kappa_{1}^{2}\right)\left(\eta_{3}^{2}+\kappa_{3}^{2}\right) \leq \widetilde{H} .
$$

Any $M$ of degree $\geq 7$ contains at least one further factor $\eta_{\nu}$ or $\kappa_{\nu}$ and contributes therefore no more than $O\left(\widetilde{H}\left|q_{\nu}\right|\right)$.

Proof of Theorems $A$ and $B$. As the Taylor series of $H(1, q)$ and $Q(1, q)$ converge for $\left|q_{\nu}\right|<1 / 3$, Lemma 6 gives $\sum_{n \geq 7} H_{n} \ll \delta \widetilde{H}, \sum_{n \geq 7} Q_{n} \ll \delta \widetilde{Q}$ if $\left|q_{\nu}\right| \leq \delta$. The two theorems now follow with the aid of Lemma 5 .

Acknowledgments. We would like to express our thanks to the colleagues and friends who, while comforting us on our English, still had a wealth of valuable suggestions.

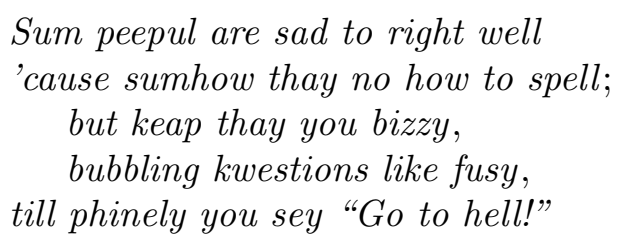

\section{References}

[1] J.-R. Chen, The lattice points in a circle, Sci. Sinica 12 (1963), 633-649.

[2] L.-K. Hua, The lattice points in a circle, Quart. J. Math. Oxford Ser. 13 (1942), $18-29$.

[3] V. Jarník, Zentralblatt für Mathematik und ihre Grenzgebiete 10 (1935), 104. 
[4] E. Krätzel, Lattice Points, Kluwer Acad. Publ., Dordrecht, 1988.

[5] S.-H. Min, On the order of $\zeta\left(\frac{1}{2}+i t\right)$, Trans. Amer. Math. Soc. 65 (1949), 448-472.

[6] H.-E. Richert, Verschärfung der Abschätzung beim Dirichletschen Teilerproblem, Math. Z. 58 (1953), 204-218.

[7] E. C. Titchmarsh, On Epstein's zeta-function, Proc. London Math. Soc. (2) 36 (1934), 485-500.

[8] -, The lattice-points in a circle, ibid. (2) 38 (1935), 96-115; Corrigendum, ibid., 555 .

[9] - On the order of $\zeta\left(\frac{1}{2}+i t\right)$, Quart. J. Math. Oxford Ser. 13 (1942), 11-17.

[10] A. Walfisz, Zentralblatt für Mathematik und ihre Grenzgebiete 8 (1934), 301.

Universität Ulm

Helmholtzstraße 18

D-89069 Ulm, Germany

E-mail: vorhauer@mathematik.uni-ulm.de wirsing@mathematik.uni-ulm.de 